\title{
The Elevated Endogenous Reactive Oxygen Species Contribute to the Sensitivity of the Amphotericin B-Resistant Isolate of Aspergillus flavus to Triazoles and Echinocandins
}

\section{Tianyu Liang, Wei Chen, Xinyu Yang, Qiqi Wang, Zhe Wan, Ruoyu Li and Wei Liu*}

Department of Dermatology and Venerology, Peking University First Hospital, National Clinical Research Center for Skin and Immune Diseases, Research Center for Medical Mycology, Peking University, Beijing Key Laboratory of Molecular Diagnosis on Dermatoses, Beijing, China

Aspergillus flavus has been frequently reported as the second cause of invasive aspergillosis (IA), as well as the leading cause in certain tropical countries. Amphotericin $B(A M B)$ is a clinically important therapy option for a range of invasive fungal infections

Edited by:

Ying-Chun Xu,

Peking Union Medical College

Hospital (CAMS), China

Reviewed by:

Jata Shankar,

Jaypee University of Information

Technology, India

Stéphane Ranque,

Aix-Marseille Université, France

*Correspondence:

Wei Liu

liuwei@bjmu.edu.cn

Specialty section:

This article was submitted to

Antimicrobials, Resistance

and Chemotherapy,

a section of the journal

Frontiers in Microbiology

Received: 15 March 2021

Accepted: 13 July 2021

Published: 03 August 2021

Citation:

Liang T, Chen W, Yang X

Wang $Q$, Wan Z, Li R and Liu W (2021) The Elevated Endogenous

Reactive Oxygen Species Contribute to the Sensitivity of the Amphotericin

B-Resistant Isolate of Aspergillus

flavus to Triazoles and Echinocandins.

Front. Microbiol. 12:680749.

doi: 10.3389/fmicb.2021.680749 including invasive aspergillosis, and in vitro resistance to $\mathrm{AMB}$ was associated with poor outcomes in IA patients treated with AMB. Compared with the AMB-susceptible isolates of $A$. terreus, the AMB-resistant isolates of $A$. terreus showed a lower level of AMBinduced endogenous reactive oxygen species (ROS), which was an important cause of $\mathrm{AMB}$ resistance. In this study, we obtained one $\mathrm{AMB}$-resistant isolate of $A$. flavus, with an AMB MIC of $32 \mu \mathrm{g} / \mathrm{mL}$, which was sensitive to triazoles and echinocandins. This isolate presented elevated endogenous ROS levels, which strongly suggested that no contribution of decreased AMB-induced endogenous ROS for AMB-resistance, opposite to those observed in A. terreus. Further, we confirmed that the elevated endogenous ROS contributed to the sensitivity of the AMB-resistant $A$. flavus isolate to triazoles and echinocandins. Further investigation is needed to elucidate the causes of elevated endogenous ROS and the resistance mechanism to $\mathrm{AMB}$ in A. flavus.

Keywords: Aspergillus flavus, amphotericin B, reactive oxygen species, triazoles, echinocandins

\section{INTRODUCTION}

Invasive aspergillosis (IA) is an important opportunistic fungal infection caused by Aspergillus with high mortality rates. Over the past few decades, the incidence of IA has been rising with the increasing number of immunosuppressed patients (Brown et al., 2012). Aspergillus flavus has been frequently reported as the second leading cause of IA, as well as the leading cause in certain tropical countries (Rudramurthy et al., 2019).

At present, there are three main classes of antifungal drugs used for the treatment of IA (Perlin et al., 2017): (i) polyenes, such as amphotericin B (AMB); (ii) triazoles, such as itraconazole (ITC), voriconazole (VRC) and posaconazole (POS); and (iii) echinocandins, such as caspofungin (CAS) and micafungin (MFG). Among them, AMB stood out due to its broad activity spectrum and less likely developed drug resistance. AMB has been a clinically important therapy option for a range of 
invasive fungal diseases, including IA, since it was first approved in the 1950s (Perlin et al., 2017). Although dose-dependent toxic side effects, such as nephrotoxicity, limit the use of AMB, the lipid formulations of AMB with equal antifungal activity were therefore developed to reduce these toxicity issues (Stone et al., 2016; Grazziotin et al., 2018). Although AMB resistance is rare, the pathogenic $A$. terreus is intrinsically resistant to AMB (Vaezi et al., 2018) and the reports of the AMB-resistant A. fumigatus and A. flavus were also notable (Ashu et al., 2018; Rudramurthy et al., 2019). Moreover, in vitro resistance to AMB was associated with poor outcomes in IA patients treated with AMB (Hadrich et al., 2012). Therefore, it is important to elucidate the mechanisms of $\mathrm{AMB}$ resistance.

Until now, the mode of antifungal action of AMB has not been well understood, and the mechanisms of $\mathrm{AMB}$ resistance also need to be elucidated. In addition to binding to ergosterol directly (Gray et al., 2012) and forming ion channels (Kristanc et al., 2019) thereby disrupting the structural integrity of cell membranes, several studies have highlighted that AMB exert antifungal activity by inducing endogenous reactive oxygen species (ROS) production, therefore resulting in oxidative damage and fungal cell death (Belenky et al., 2013; Mesa-Arango et al., 2014; Shekhova et al., 2017). Studies on A. terreus, intrinsic resistance to $\mathrm{AMB}$, revealed that the AMB-resistant clinical isolates of A. terreus could handle better with $\mathrm{AMB}$-induced oxidative stress and thus showed a lower level of AMB-induced endogenous ROS, compared with AMB-susceptible clinical isolates of $A$. terreus (Blatzer et al., 2015; Jukic et al., 2017). These studies are important for understanding the mechanisms of resistance to AMB in pathogenic fungi, including A. flavus.

In this study, we screened the susceptibility of clinical isolates of $A$. flavus to AMB by using the broth microdilution method according to the Clinical and Laboratory Standards Institute (CLSI) M38-A3 guideline. From 117 clinical isolates of A. flavus, we obtained an AMB-resistant A. flavus isolate. To investigate the role of ROS in AMB resistance in this isolate, the sensitivity to oxidative stress and endogenous ROS levels with or without exposure to $\mathrm{AMB}$ were determined. Meanwhile, the expression level and activities of enzymes involved in ROS detoxification were also investigated. In addition, the endogenous ROS levels induced by triazoles and echinocandins were also measured, and the ROS scavenger $\mathrm{N}$-acetylcysteine (NAC) was used to investigate the effect of ROS levels on in vitro antifungal susceptibility in AMB-resistant A. flavus isolate.

\section{MATERIALS AND METHODS}

\section{Antifungal Susceptibility Testing}

Antifungal susceptibility testing by the broth microdilution method was performed according to the recommendations of the CLSI M38-A3 document (Clinical and Laboratory Standards Institute (CLSI)., 2017), and the tested drugs included were ITC, VRC, POS, CAS, MFG (all from Harveybio Gene Technology Co., Ltd., Beijing, China) and AMB (North China Pharmaceutical Co., Ltd., Shijiazhuang, China). Briefly, antifungal drugs were dispensed into 96-well plates at final concentration ranges of $0.0625-32 \mu \mathrm{g} / \mathrm{mL}$ for $\mathrm{AMB}, 0.0313-16 \mu \mathrm{g} / \mathrm{mL}$ for ITC, VRC, and POS, and $0.008-4 \mu \mathrm{g} / \mathrm{mL}$ for CAS and MFG. All isolates of $A$. flavus were subcultured on potato dextrose agar (PDA) at $35^{\circ} \mathrm{C}$ for 3 to 7 days to yield good conidiation. Conidia were harvested by slightly scraping the surface of the A. flavus colonies with a sterile cotton swab and suspending the colonies in sterile saline solution with $0.05 \%$ Tween-20. Heavy particles were allowed to settle for $5 \mathrm{~min}$, after which the upper homogenous suspensions were used as inoculum suspensions. Inoculum suspensions were diluted in RPMI 1640 medium at a final concentration of $1 \times 10^{4} \mathrm{CFU} / \mathrm{mL}$, as determined by a hemocytometer, and transferred into 96well plates containing drug dilutions. The 96-well plates were incubated at $35^{\circ} \mathrm{C}$ and examined visually for MIC (after $48 \mathrm{~h}$ ) and MEC (after $24 \mathrm{~h}$ ) determinations. The MIC endpoints for $\mathrm{AMB}$ and triazoles were determined as the lowest drug concentration that resulted in a $100 \%$ reduction in growth compared with that of the drug-free controls. The MEC endpoints for echinocandins were determined as the lowest drug concentration that led to the growth of small, rounded, compact hyphal forms compared with the hyphal growth seen in the growth control well.

Antifungal susceptibility testing by E-test was performed according to the manufacturer's instructions. Briefly, inoculum suspensions at a final concentration of $1 \times 10^{6} \mathrm{CFU} / \mathrm{mL}$ were inoculated on the entire surface of each $90-\mathrm{mm}$ plate containing $25 \mathrm{~mL}$ of RPMI 1640 medium (in the presence or absence of $15 \mathrm{mM}$ NAC) with a sterile cotton swab. The E-test strips (Autobio, Zhengzhou, China) were placed on the center of the plate and incubated at $35^{\circ} \mathrm{C}$. The MIC or MEC (for CAS only) was determined from the inhibition ellipse that intersected the scale on the strip after $48 \mathrm{~h}$.

Antifungal susceptibility testing by disk diffusion was performed on non-supplemented Muller-Hinton (NMH) agar refer to the method described previously (Qiao et al., 2007). When necessary, a $15 \mathrm{mM}$ concentration of the antioxidant NAC was dissolved in $\mathrm{NMH}$ medium. Disks prepared in-house of AMB $(50 \mu \mathrm{g})$, ITC $(10 \mu \mathrm{g})$, VRC (5 $\mu \mathrm{g})$, POS $(5 \mu \mathrm{g})$, CAS $(5 \mu \mathrm{g})$, and MFG $(5 \mu \mathrm{g})$ were placed onto the surface of the inoculated (the same method as described in the E-test) $\mathrm{NMH}$ plate. The plates were incubated at $35^{\circ} \mathrm{C}$, and the inhibition zone diameter was determined after $48 \mathrm{~h}$.

\section{Testing of Sensitivity to Oxidative Stress}

Based on the reported studies that AMB-resistant $A$. terreus can handle oxidative stress better, two $A$. terreus clinical isolates with different susceptibilities to AMB, the AMB-susceptible A. terreus isolate BMU09523 (MIC $=2 \mu \mathrm{g} / \mathrm{ml})$ and the AMB-resistant A. terreus isolate BMU05143 (MIC $=8 \mu \mathrm{g} / \mathrm{mL})$ were included for comparison. We tested the sensitivity of $A$. flavus isolates and $A$. terreus isolates to $\mathrm{H}_{2} \mathrm{O}_{2}$ by spot assay. $\mathrm{H}_{2} \mathrm{O}_{2}$ at a final concentration of $1 \mathrm{mM}$ was supplemented in PDA medium with or without antifungal drugs. Serially diluted inoculum suspensions $(2 \mu \mathrm{l})$ were spotted onto PDA plates and incubated at $35^{\circ} \mathrm{C}$ for $48 \mathrm{~h}$. 
TABLE 1 | Primers used in this study.

\begin{tabular}{|c|c|c|}
\hline Locus tag & Gene ID & Sequence $\left(5^{\prime}-3^{\prime}\right)$ \\
\hline \multirow[t]{2}{*}{ AFLA_056170 } & catA & TGTGAAGGTCGCTACGTCTG \\
\hline & & ACGCTTGTAGTTCCGATGCT \\
\hline \multirow[t]{2}{*}{ AFLA_100250 } & cat & CGAGACACTGGCTCATITCA \\
\hline & & ACCGGTGGTACTGATTCTGC \\
\hline \multirow[t]{2}{*}{ AFLA_090690 } & cat1 & CTCCAAGCTCGTCAAGTTCC \\
\hline & & GATCGAAGCCAAACTTCAGC \\
\hline \multirow[t]{2}{*}{ AFLA_122110 } & cat2 & TCAATCAGATGGAGCCTGTG \\
\hline & & GCCGGGTAGTAAACACTCCA \\
\hline \multirow[t]{2}{*}{ AFLA_096210 } & cat3 & ATAATGTCGGTCGCAAGTCC \\
\hline & & CTTCGCATACTCTGGTGCAA \\
\hline \multirow[t]{2}{*}{ AFLA_034380 } & cat4 & TGAGACTCTCGCCCATTTCT \\
\hline & & CCCAGTCCAAGTTACCCTCA \\
\hline \multirow[t]{2}{*}{ AFLA_044930 } & sod1 & ATTGAAGGCTACGGTGTTGG \\
\hline & & СССTCTIGСTCTTCGACAC \\
\hline \multirow[t]{2}{*}{ AFLA_068080 } & sod2 & GCGACATAAGCGGAAAACAT \\
\hline & & GTCTTCCTTCGCCTCтTCCT \\
\hline \multirow[t]{2}{*}{ AFLA_033420 } & sod3 & ATGGAAATCCACCACCAAAA \\
\hline & & AGAGGGAGTGGTTGATGTGG \\
\hline \multirow[t]{2}{*}{ AFLA_027580 } & sod4 & АСTCTGCCTGACCTGGCTTA \\
\hline & & AGTGGTGATGTCCTCCTTGG \\
\hline \multirow[t]{2}{*}{ AFLA_088150 } & sod5 & GAGATGGCCTCCGTATTCAA \\
\hline & & САТСААТССТТСССТСТССА \\
\hline \multirow[t]{2}{*}{ AFLA_099000 } & sod6 & CACCAGTTCGGTGACAACAC \\
\hline & & GTACGGCCAAGTACGCTCTC \\
\hline
\end{tabular}

\section{Measurement of Endogenous ROS Level}

The endogenous ROS level of the AMB-resistant A. flavus isolate was determined by $2^{\prime}, 7^{\prime}$-dichlorofluorescin diacetate (DCF-DA) as previously described (Shekhova et al., 2017). In brief, $100 \mu \mathrm{L}$ conidial suspensions at a concentration of $1 \times 10^{4} \mathrm{CFU} / \mathrm{mL}$ were dispensed into flat-bottom 96 -well plates, followed by incubation at $37^{\circ} \mathrm{C}$ for $18 \mathrm{~h}$. After a washing step with phosphate buffered saline (PBS), the cells were stained with $10 \mu \mathrm{M}$ DCF-DA for $30 \mathrm{~min}$ at $37^{\circ} \mathrm{C}$ in the dark. After washing with PBS, different antifungal drugs prepared in PBS were added to the cells. PBS was used as a negative control and $2 \mathrm{mM} \mathrm{H}_{2} \mathrm{O}_{2}$ was used as a positive control. The fluorescence intensity (excitation filter at $485 \mathrm{~nm}$ and emission filter at $530 \mathrm{~nm}$ ) was measured by a microtiter plate reader (Infinite 200 Pro, Tecan, Switzerland) and observed under fluorescence microscope simultaneously at $37^{\circ} \mathrm{C}$. The maximum fluorescence intensity observed after $2 \mathrm{~h}$ of incubation with drugs was recorded as a reference to the endogenous ROS level.

\section{Assessment of Genes Encoding Enzymes Involved in ROS Detoxification by RT-qPCR}

To identify homologs of enzymes involved in ROS detoxification in A. flavus, the amino acid sequences of catalases (CATs) and superoxide dismutases (SODs) in A. fumigatus and A. terreus (Jukic et al., 2017) were used as queries to perform BLASTP analysis $^{1}$ in the genome database of A. flavus. The primers

\footnotetext{
${ }^{1}$ https://blast.ncbi.nlm.nih.gov/Blast.cgi
}

used in Reverse transcription-quantitative PCR (RT-qPCR) were designed on the Primer3Plus ${ }^{2}$. The identified putative genes encoding enzymes involved in ROS detoxification in A. flavus and the primers are listed in Table $\mathbf{1 .}$

For assessment of expression of genes encoding enzymes involved in ROS detoxification, a total of $1 \times 10^{6} \mathrm{CFU}$ conidia were dispensed into Aspergillus minimal medium followed by incubation at $37^{\circ} \mathrm{C}$ for $18 \mathrm{~h}$ on an orbital shaker at $200 \mathrm{rpm}$. Different antifungal drugs prepared in PBS or PBS were added at $37^{\circ} \mathrm{C}$ for an additional $2 \mathrm{~h}$ on an orbital shaker at $200 \mathrm{rpm}$. Then the hyphae were harvested and total RNA was extracted following liquid nitrogen crush using TRIzol reagent (Invitrogen). cDNA was synthesized using an Advantage RT-for-PCR kit (Clontech) according to the manufacturer's instructions. RT-qPCR was performed on an Applied Biosystems ViiA7 Real-Time PCR system using SYBR green reagent (Applied Biosystems). The cycling conditions were as follows: a 10-min initial denaturation at $95^{\circ} \mathrm{C}$, followed by 40 cycles of denaturation at $95^{\circ} \mathrm{C}$ for $15 \mathrm{~s}$, and annealing/extension at $60^{\circ} \mathrm{C}$ for $10 \mathrm{~s}$. Changes in gene expression were calculated using the $2^{-\Delta \Delta C t}$ method (Schmittgen and Livak, 2008). All experiments were performed in triplicate from biological triplicates.

\section{Determination of CAT, SOD and GSH-Px Activity}

To determine CAT, SOD and glutathione peroxidase (GSH-Px) activity of the A. flavus isolate, the hyphae were harvested as conditions described in the RT-qPCR assay. The enzyme activity was determined using the CAT activity assay kit (Abcam), the SOD activity assay kit (Abcam), and GSH-Px activity assay kit (Abcam) separately according to the manufacturer's instructions. The relative enzyme activities (\%) were calculated relative to those of A. flavus NRRL3357 under basal conditions.

\section{Statistical Analysis}

Experiments were performed at least three independent biological replicates. A Welch two-sample $t$ test was used for significance testing of two groups. $P$-values $<0.05$ were considered statistically significant.

\section{RESULTS}

\section{The AMB-Resistant Isolate of $A$. flavus Showed Sensitivity to Triazoles and Echinocandins}

From 117 clinical isolates of A. flavus, we obtained one AMBresistant isolate of A. flavus, named BMU09525, with an AMB MIC of $32 \mu \mathrm{g} / \mathrm{mL}$. The MICs of ITC, VRC, and POS against A. flavus BMU09525 were 0.06, 0.25, and $0.03 \mu \mathrm{g} / \mathrm{mL}$, respectively. The MECs of CAS and MFG for A. flavus BMU09525 were both $0.008 \mu \mathrm{g} / \mathrm{mL}$ (Table 2). The results showed that the AMB-resistant isolate of A. flavus BMU09525 was sensitive to triazoles (ITC, VRC, POS) and echinocandins

\footnotetext{
${ }^{2}$ https://primer3plus.com/cgi-bin/dev/primer3plus.cgi
} 
TABLE 2 | Antifungal susceptibility testing by the broth microdilution method $(\mu \mathrm{g} / \mathrm{mL})$, E-test $(\mu \mathrm{g} / \mathrm{mL})$, and disk diffusion $(\mathrm{mm})$.

\begin{tabular}{|c|c|c|c|c|}
\hline & Methods & NAC & NRRL3357 & BMU09525 \\
\hline \multirow[t]{5}{*}{ AMB } & ВММ & - & 2 & 32 \\
\hline & E-test & - & 2 & $>32$ \\
\hline & & + & 2 & $>32$ \\
\hline & DD & - & 15 & 0 \\
\hline & & + & 12 & 0 \\
\hline \multirow[t]{5}{*}{ ITC } & ВММ & - & 0.25 & 0.06 \\
\hline & E-test & - & 2 & 0.25 \\
\hline & & + & 4 & 0.75 \\
\hline & DD & - & 33 & 47 \\
\hline & & + & 29 & 34 \\
\hline \multirow[t]{5}{*}{ VRC } & ВMМ & - & 0.5 & 0.25 \\
\hline & E-test & - & 0.032 & 0.024 \\
\hline & & + & 0.064 & 0.064 \\
\hline & DD & - & 40 & 51 \\
\hline & & + & 34 & 44 \\
\hline \multirow[t]{5}{*}{ POS } & ВMM & - & 0.125 & 0.03 \\
\hline & E-test & - & NA & NA \\
\hline & & + & NA & NA \\
\hline & DD & - & 32 & 46 \\
\hline & & + & 33 & 30 \\
\hline \multirow[t]{5}{*}{ CAS } & ВММ & - & 0.03 & 0.008 \\
\hline & E-test & - & 0.25 & 0.064 \\
\hline & & + & 0.5 & 0.25 \\
\hline & DD & - & 27 & 25 \\
\hline & & + & 23 & 22 \\
\hline \multirow[t]{5}{*}{ MFG } & BMM & - & 0.03 & 0.008 \\
\hline & E-test & - & NA & NA \\
\hline & & + & NA & NA \\
\hline & DD & - & 40 & 36 \\
\hline & & + & 40 & 28 \\
\hline
\end{tabular}

BMM, broth microdilution method; DD, disk diffusion; NA, E-test strips were not available.

(CAS, MFG). Similar results were obtained by the disk diffusion method and E-test (Figure $\mathbf{1}$ and Table 2). Interestingly, when testing echinocandins against the A. flavus strain NRRL3357, microcolonies within a well-defined zone of inhibition could be seen, while testing echinocandins against AMB-resistant isolate of A. flavus, the inhibition ellipse of E-test strip (CAS) or the inhibition zone of the disk (CAS and MFG) was as clean as that seen in triazoles against the AMB-resistant A. flavus isolate, suggesting that echinocandins may exert a fungicidal effect, instead of a fungistatic effect, against the AMB-resistant isolate of A. flavus.

\section{The AMB-Resistant Isolate of $A$. flavus Showed Hypersensitivity to Oxidative Stress, Opposite to That Observed in the AMB-Resistant $A$. terreus}

The AMB-resistant $A$. terreus isolate showed better tolerance to $\mathrm{H}_{2} \mathrm{O}_{2}$ than the AMB-susceptible A. terreus isolates (Figure 2A), consistent with previous studies on A. terreus. Surprisingly, when exposed to $\mathrm{H}_{2} \mathrm{O}_{2}$, the AMB-resistant A. flavus isolate showed more obvious growth inhibition than the A. flavus strain NRRL3357 (Figure 2B). When $\mathrm{H}_{2} \mathrm{O}_{2}$ was combined with antifungals, the A. flavus strain NRRL3357 showed merely slight growth inhibition compared to that using antifungals alone. However, $\mathrm{H}_{2} \mathrm{O}_{2}$ could significantly enhance the activity of antifungals against the AMB-resistant A. flavus isolate (Figure 2B). The above results indicated that the AMB-resistant A. flavus isolate was hypersensitive to oxidative stress, in contrast to the case reported for $A$. terreus. In addition, a decreased growth rate of the AMB-resistant A. flavus isolate could be observed compared to the A. flavus strain NRRL3357 (Figure 2B).

\section{The AMB-Resistant Isolate of $A$. flavus Showed Elevated Basal Endogenous ROS}

The basal endogenous ROS level of the AMB-resistant A. terreus isolate was comparable to that of the AMB-susceptible A. terreus isolate, while the basal endogenous ROS level of the AMBresistant $A$. flavus isolate was significantly higher than that of both the A. flavus NRRL3357 and A. terreus (Figures 3, 4). The elevated basal endogenous ROS in AMB-resistant $A$. flavus isolate suggested that the mechanisms of AMB resistance in AMBresistant $A$. flavus isolate may differ from those mediating AMB resistance in $A$. terreus.

\section{The AMB-Resistant Isolate of $A$. flavus Showed Comparable ROS Detoxification Enzyme Activities}

Because the AMB-resistant A. flavus isolate showed elevated basal endogenous ROS level and was sensitive to oxidative stress, we further tested the expression level of sod and cat genes in A. flavus. A total of 6 sod and 6 cat genes were investigated in A. flavus (Figure 5 and Table 1). And the ROS detoxification enzyme activities, including CAT, SOD, and GSH-Px (Figure 6), were also measured.

Except for cat gene, the basal level of cat $A$, cat 1, cat2, cat 3 , and cat4 in the AMB-resistant A. flavus isolate were mildly higher than that of the A. flavus NRRL3357. Upon $\mathrm{H}_{2} \mathrm{O}_{2}$ exposure, cat $A$, cat 2 and cat 3 expression level increased in both the AMBresistant A. flavus isolate and the A. flavus NRRL3357, while no significant changes of cat, cat1, and cat 4 in both two strains. However, a different picture was observed in sod genes expression level. In the AMB-resistant A. flavus isolate, only sod 2 showed a higher basal level than that of the A. flavus NRRL3357, while the basal expression level of sod1, sod5 and sod6 were less than that of the A. flavus NRRL3357. The basal expression levels of both sod3 and sod4 did not differ between these two A. flavus strains. After exposure to $\mathrm{H}_{2} \mathrm{O}_{2}$, sod1, sod2, sod5, and $\operatorname{sod} 6$ showed a significant increase in the AMB-resistant A. flavus isolate, while only sod 2 showed elevated transcript level in the A. flavus NRRL3357.

Next, we tested the enzyme activities of CAT, SOD, and GSH-Px using the commercially available kits (Figure 5). The basal enzyme activities of CAT, SOD or GSH-Px in the AMBresistant A. flavus isolate were not significantly different from those in the A. flavus NRRL3357. After exposure to $\mathrm{H}_{2} \mathrm{O}_{2}$, 


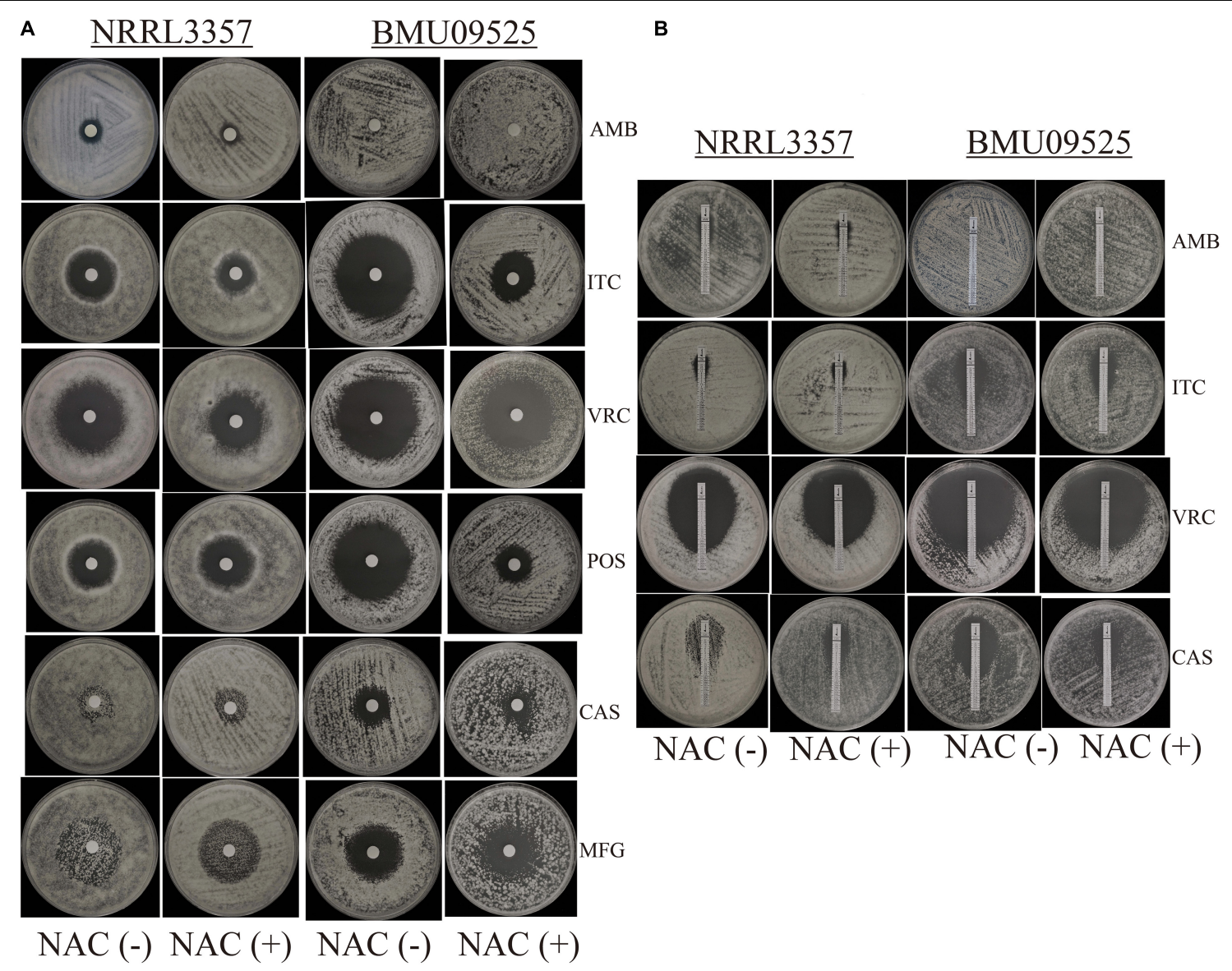

FIGURE 1 | In vitro antifungal susceptibility testing. (A) In vitro antifungal susceptibility testing determined by disk diffusion. The antioxidant N-Acetylcysteine (NAC) was added at a concentration of $15 \mathrm{mM}$. Disks of AMB $(50 \mu \mathrm{g})$, ITC $(10 \mu \mathrm{g})$, VRC $(5 \mu \mathrm{g})$, POS $(5 \mu \mathrm{g})$, CAS $(5 \mu \mathrm{g})$, and MFG $(5 \mu \mathrm{g})$ were placed onto the NMH medium. Plates were incubated at $35^{\circ} \mathrm{C}$ for $48 \mathrm{~h}$. (B) In vitro antifungal susceptibility testing determined by E-test. Plates were incubated at $35^{\circ} \mathrm{C}$ for $48 \mathrm{~h}$.

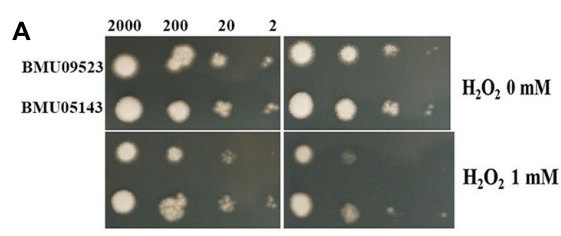

B

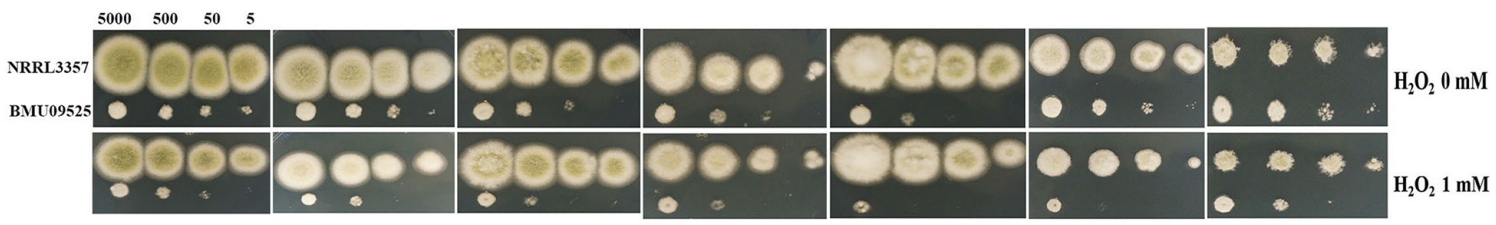

FIGURE 2 | The sensitivity to $\mathrm{H}_{2} \mathrm{O}_{2}$ determined by spot assay. Hydrogen peroxide $\left(\mathrm{H}_{2} \mathrm{O}_{2}\right)$ at a concentration of $1 \mathrm{mM}$ was supplemented in PDA medium with or without antifungal drugs. (A) A total of 2, 20, 200, and $2 \times 10^{3}$ conidia of $A$. terreus were spotted onto PDA medium, respectively. (B) A total of 5, 50, 500, and $5 \times 10^{3}$ conidia of $A$. flavus were spotted onto PDA medium, respectively. Plates were incubated at $35^{\circ} \mathrm{C}$ and documented after $48 \mathrm{~h}$.

the activities of all these enzymes showed an increase but remained comparable between the two strains. Nevertheless, the increase of enzyme activities of CAT and GSH-Px in the A. flavus NRRL3357 was more significant than that in the AMB-resistant A. flavus isolate, which showed only a mild increase. 

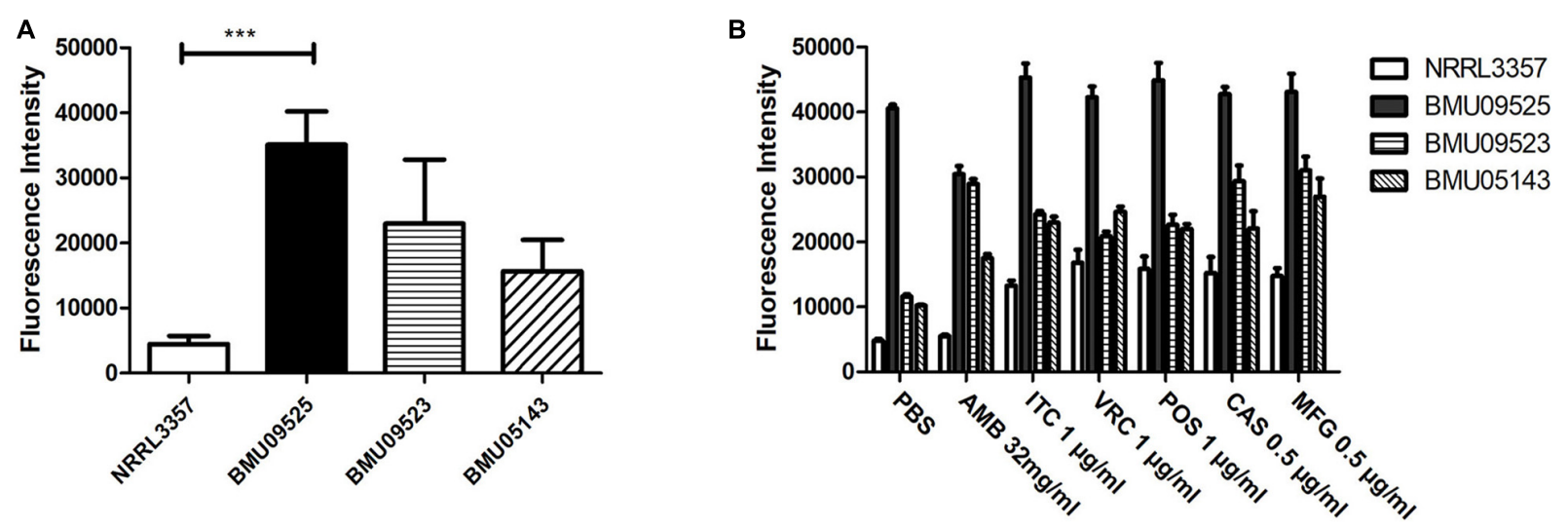

FIGURE 3 | Level of endogenous ROS determined by DCF-DA assay. (A) The level of basal endogenous ROS. (B) The level of endogenous ROS induced by antifungals. Conidial were incubated at $37^{\circ} \mathrm{C}$ for $18 \mathrm{~h}$ before stained with $10 \mu \mathrm{M}$ DCF-DA for 30 min at $37^{\circ} \mathrm{C}$ in the dark. After a wash step, different antifungal drugs prepared in PBS were added to the cells. The fluorescence intensity peak was observed after $2 \mathrm{~h}$ of incubation with drugs.

Overall, these results indicate that the basal activities of the enzyme involved in ROS detoxification in the AMB-resistant A. flavus isolate were comparable to those in the A. flavus NRRL3357, despite the different expression levels were observed. And the elevated basal endogenous ROS level in the AMBresistant $A$. flavus isolate may not be related to abnormal ROS detoxification enzyme activities. When exposure to $\mathrm{H}_{2} \mathrm{O}_{2}$, however, the less remarkable increase in enzyme activities of CAT and GSH-Px in the AMB-resistant A. flavus isolate may result in hypersensitivity to oxidative stress.

\section{Triazoles and Echinocandins, Instead of AMB, Could Induce the Production of Endogenous ROS in the AMB-Resistant Isolate of $A$. flavus}

To further investigate the relationships between ROS levels and antifungal susceptibilities in the AMB-resistant A. flavus isolate, the endogenous ROS levels induced by antifungals were determined (Figures 3, 4). With exposure to AMB, the endogenous ROS level of the AMB-susceptible A. terreus isolate was significantly increased, while that in the AMB-resistant A. terreus isolate increased slightly, consistent with the literature reports on $A$. terreus. Surprisingly, the endogenous ROS level of the A. flavus strain NRRL3357 increased slightly, while the endogenous ROS level of the AMB-resistant A. flavus isolate even showed a minor decrease despite the ROS level in the AMBresistant $A$. flavus isolate being significantly higher than that of the A. flavus strain NRRL3357. With exposure to triazoles and echinocandins, the endogenous ROS levels of both $A$. terreus and $A$. flavus isolates increased to different degrees. These results strongly suggested no contribution of AMB-induced endogenous ROS to AMB resistance in the AMB-resistant A. flavus isolate, in contrast to the situation observed in A. terreus. In addition, the elevated basal endogenous ROS in the AMBresistant $A$. flavus isolate might result in its sensitivity to triazoles and echinocandins.

\section{ROS Elimination by the Antioxidant NAC Decreased the Sensitivity of the AMB-Resistant $A$. flavus Isolate to Triazoles and Echinocandins}

The antioxidant NAC can act as a non-specific sulfhydryl donor to scavenge intracellular ROS. Adding NAC to the medium did not show any impact on the susceptibility to $\mathrm{AMB}$, corresponding to the result that no $\mathrm{AMB}$-induced ROS production in the AMB-resistant A. flavus isolate (Figure $\mathbf{1 A}$ and Table 2). However, adding NAC to the medium reduced the inhibition zone of triazoles and echinocandins, indicating the decreased susceptibility of AMB-resistant A. flavus isolate to triazoles and echinocandins (Figure 1A and Table 2). Consistent results were obtained by E-test (Figure 1B and Table 2), which showed that NAC increased the MICs of triazoles and the MECs of echinocandins obtained from E-test strips. The above results confirmed our assumption that the increased level of endogenous ROS contributes to the sensitivity of the AMB-resistant A. flavus isolate to triazoles and echinocandins. Interestingly, the addition of NAC decreased the susceptibility of the A. flavus strain NRRL3357 to ITC, VRC, and CAS, similar to the AMBresistant $A$. flavus isolate. Although adding NAC decreased the susceptibility of the A. flavus strain NRRL3357 to AMB, the addition of NAC did not change its susceptibility to POS or MFG, as no alteration in the inhibition zone diameter was observed by disk diffusion.

\section{DISCUSSION}

Several studies have reported that AMB can induce endogenous ROS production as its mode of action (Belenky et al., 2013; MesaArango et al., 2014; Shekhova et al., 2017). Due to the intrinsic resistance of $A$. terreus to $\mathrm{AMB}$, the impact of endogenous ROS production on the susceptibility of this species to AMB has been closely studied (Posch et al., 2018). Compared with AMB-susceptible A. terreus isolates, AMB-resistant A. terreus 

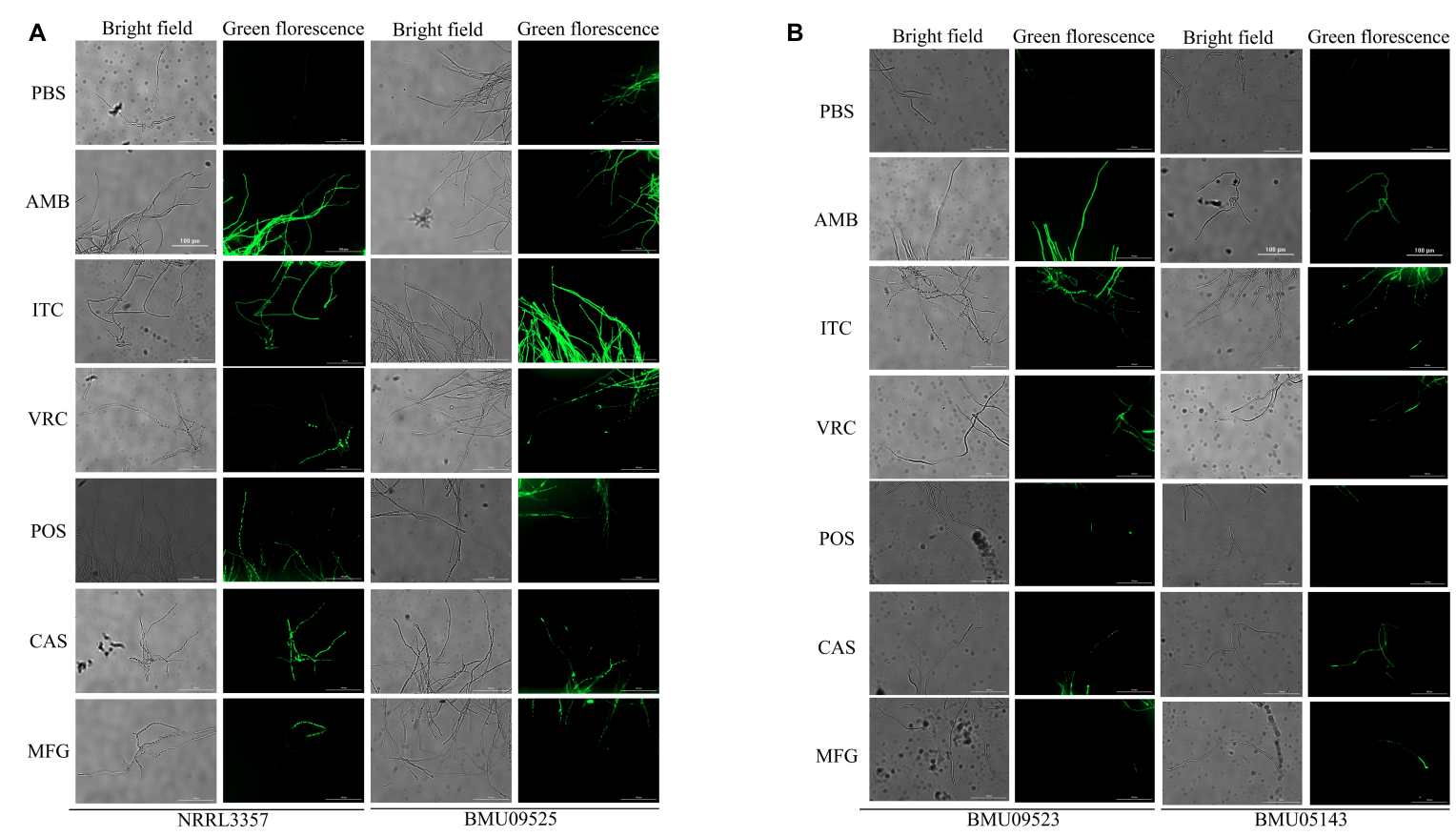

FIGURE 4 | Fluorescence microscope images showing endogenous ROS in (A) A. flavus. (B) A. terreus. Conidial were incubated at $37^{\circ} \mathrm{C}$ for $18 \mathrm{~h}$ before stained with $10 \mu \mathrm{M}$ DCF-DA for $30 \mathrm{~min}$ at $37^{\circ} \mathrm{C}$ in the dark. After a wash step, Fluorescence images were recorded after $2 \mathrm{~h}$ of incubation with drugs. AMB (32 $\left.\mu \mathrm{g} / \mathrm{ml}\right)$, ITC $(1 \mu \mathrm{g} / \mathrm{ml})$, VRC (1 $\mu \mathrm{g} / \mathrm{ml})$, POS $(1 \mu \mathrm{g} / \mathrm{ml})$, CAS $(0.5 \mu \mathrm{g} / \mathrm{ml})$, MFG $(0.5 \mu \mathrm{g} / \mathrm{ml})$.

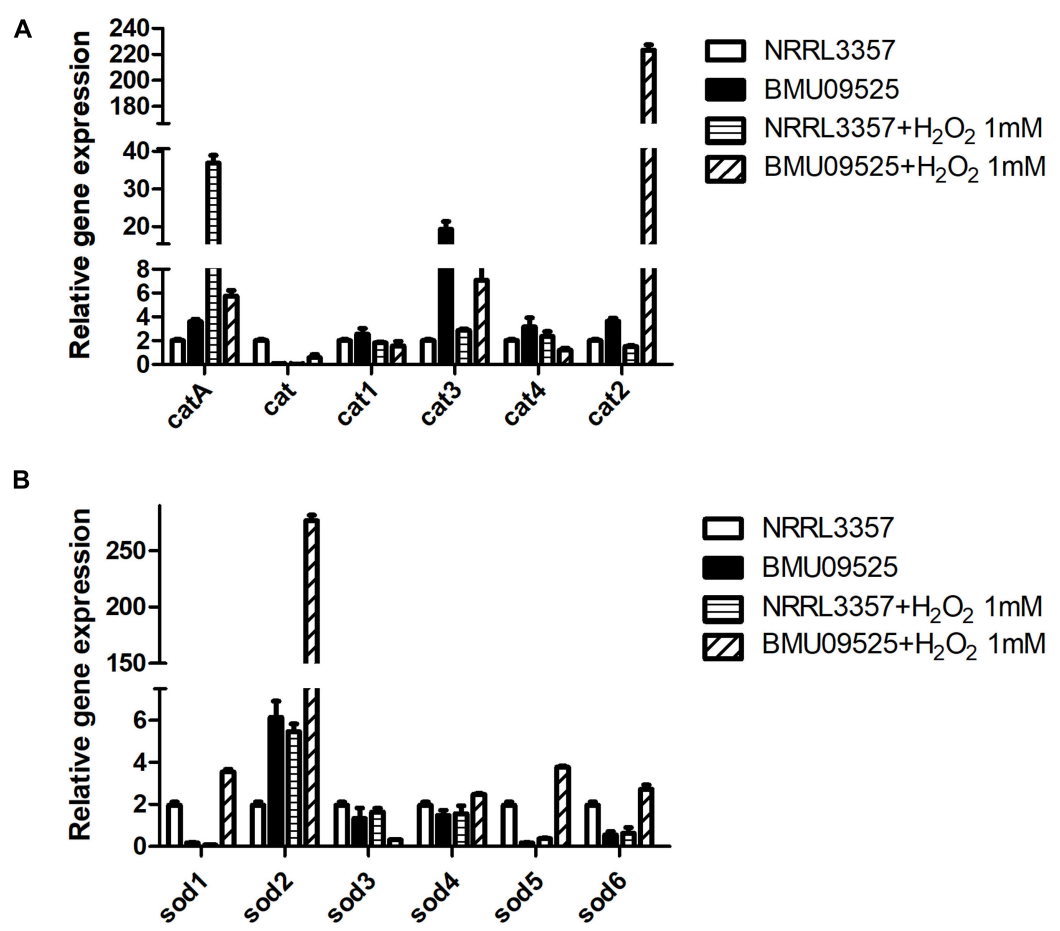

FIGURE 5 | The expression level of (A) cat genes and (B) sod genes in A. flavus. A total of $1 \times 10^{6} \mathrm{CFU}$ A. flavus conidia were preincubated in Aspergillus minimal medium for $18 \mathrm{~h}$ at $37^{\circ} \mathrm{C}$ and $200 \mathrm{rpm}$ before $\mathrm{H}_{2} \mathrm{O}_{2}(1 \mathrm{mM})$ was added for $2 \mathrm{~h}$. Then the hyphae were harvested and total RNA was extracted following liquid nitrogen crush. Gene expression was normalized to that of beta-tubulin according to the $2^{-\Delta \Delta \mathrm{Ct}}$ method. Data are presented as means \pm standard deviations for three independent experiments with technical duplicates. 


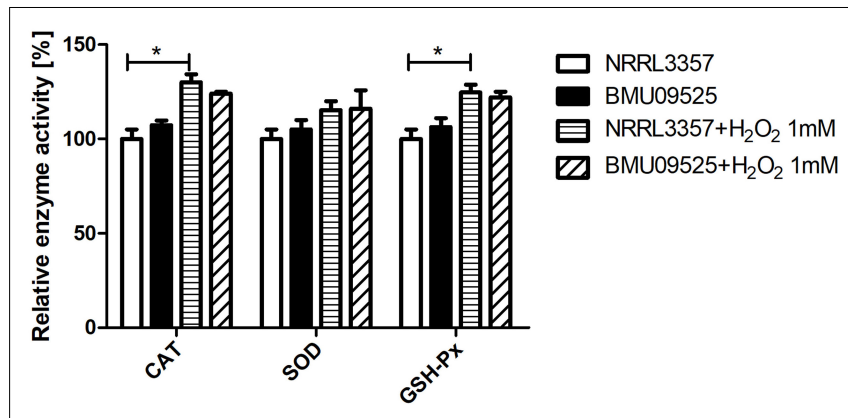

FIGURE 6 | Determination of the enzyme activities of SOD, CAT and GSH-Px. A total of $1 \times 10^{6} \mathrm{CFU} A$. flavus conidia were preincubated in Aspergillus minimal medium for $18 \mathrm{~h}$ at $37^{\circ} \mathrm{C}$ and $200 \mathrm{rpm}$ before $\mathrm{H}_{2} \mathrm{O}_{2}(1 \mathrm{mM})$ was added for $2 \mathrm{~h}$. Then the hyphae were harvested and enzyme activities were determined according to the manufacturer's instructions. The relative enzyme activities (\%) were calculated relative to those of $A$. flavus NRRL3357 under basal conditions. CAT, catalase; SOD, superoxide dismutase; GHS-Px, glutathione peroxidase.

isolates presented decreased AMB-induced ROS production in mitochondria (Blatzer et al., 2015) and higher ROS-detoxifying enzyme activity (Jukic et al., 2017). However, the AMB-resistant isolate of $A$. flavus presented an elevated level of endogenous ROS regardless of exposure to $\mathrm{AMB}$ and was hypersensitive to oxidative stress. Further studies revealed that the less remarkable increase in enzyme activities of CAT and GSH-Px may result in its hypersensitivity to oxidative stress, compared to the observations in A. terreus (Blatzer et al., 2015; Jukic et al., 2017). These results suggested that the higher activity of ROS-detoxifying enzyme did not contribute to resistance to AMB in the AMBresistant isolate of $A$. flavus. The above results indicate that the mechanisms underlying $\mathrm{AMB}$ resistance in AMB-resistant A. flavus isolate differ from those mediating $\mathrm{AMB}$ resistance in A. terreus. Although AMB resistance has not been reported to be associated with the missing ergosterol in Aspergillus spp. (Blum et al., 2008, 2013), the absence of ergosterol in Candida spp., caused by mutations in genes of the ergosterol biosynthesis (Geber et al., 1995; Sanglard et al., 2003; Martel et al., 2010a; Vincent et al., 2013; Silva et al., 2020), leading to AMB resistance and abnormal membrane structure and function, which also drastically diminished tolerance to oxidative stress (Vincent et al., 2013). These studies are consistent with the phenotypes observed in the AMB-resistant isolate of A. flavus. Further membrane sterol profile analysis is needed to elucidate its mechanisms of $\mathrm{AMB}$ resistance.

Triazoles exert antifungal effects by inhibiting sterol $14 \alpha$-demethylase (CYP51A/ERG11), which prevents ergosterol biosynthesis and causes the accumulation of toxic sterols (Martel et al., 2010b; Warrilow et al., 2010, 2019). Echinocandins target the $\beta$-1,3-glucan synthase of the fungal cell wall and inhibit the synthesis of $\beta-1,3-\mathrm{D}$ glucan on the cell wall (Perlin, 2015). In addition to the above targets, several studies have reported that triazoles (Shirazi et al., 2013; Shekhova et al., 2017; Lee and Lee, 2018) and echinocandins (Belenky et al., 2013; Hao et al., 2013; Delattin et al., 2014) are capable of inducing endogenous ROS production. The AMB-resistant A. flavus isolate was sensitive to triazoles and echinocandins, while having elevated basal endogenous ROS. Further, $\mathrm{H}_{2} \mathrm{O}_{2}$ significantly enhanced the antifungal effects of triazoles and echinocandins in vitro, showing a synergistic effect against the AMB-resistant A. flavus isolate. Thus, we hypothesized that sensitivity of AMB-resistant A. flavus isolate to triazoles and echinocandins may be caused by elevated endogenous ROS levels. The antioxidant NAC can act as a non-specific sulfhydryl donor and is widely used to scavenge intracellular ROS (Dringen and Hamprecht, 1999; Dekhuijzen, 2004). Scavenging ROS by NAC decreased the sensitivity of AMB-resistant A. flavus isolate to triazoles and echinocandins confirmed our hypothesis. Interestingly, NAC did not affect the susceptibility of A. flavus strain NRRL3357 to POS and MFG, suggesting that ROS, albeit can be induced by POS and MFG, may not be necessary in antifungal mode of action.

Reactive oxygen species are derived from oxygen and known to be of biological importance in eukaryotic cells (Sena and Chandel, 2012). Mitochondria possess the oxidative phosphorylation system, which is the major origin of ROS generation (Zorov et al., 2014). Inappropriate electron transfer reactions in mitochondrial electron transport chain can produce excessive ROS. These highly reactive and toxic ROS can cause cellular damage, ultimately resulting in cell death (Zorov et al., 2014). Correspondingly, ROS can be eliminated by multiple antioxidant enzymes in eukaryotic cells, including SOD (Lambou et al., 2010), CAT (Shibuya et al., 2006), and GSHPx (Margis et al., 2008). Studies in A. terreus (Blatzer et al., 2015; Jukic et al., 2017), the AMB-resistant A. terreus isolates exhibited distinct basal expression levels of sod and cat genes compared to the AMB-susceptible A. terreus isolates. However, the basal enzyme activities of CAT and SOD of the AMBresistant $A$. terreus isolates were already higher than that of the AMB-susceptible $A$. terreus isolate. In this study, the two A. flavus strains also exhibited distinct basal expression levels of sod and cat genes. The basal expression level of catA, cat3, cat4, and sod2 in the AMB-resistant A. flavus isolate were higher than that of the A. flavus NRRL3357, while the basal expression level of cat, sod1, sod5, and sod6 were less than that of the A. flavus NRRL3357. However, the basal enzyme activities of CAT and SOD in the AMB-resistant A. flavus isolate were comparable to those in the A. flavus NRRL3357. Taken together, although no elevated enzyme activity was observed in the AMB-resistant A. flavus isolate in the basal condition as reported in the AMB-resistant $A$. terreus, the basal enzyme activity in the AMB-resistant A. flavus isolate was still comparable to the AMB-susceptible A. terreus isolates. Therefore, it is reasonable to speculate that the elevated basal endogenous ROS level is due to increased production rather than impaired clearance in the AMB-resistant A. flavus isolate. Since mitochondria are the main site of ROS production, it is likely that the elevated endogenous ROS level in the AMB-resistant A. flavus isolate may be caused by the dysfunction mitochondrial which may lead to overproduction of ROS. Also, the slowed growth observed in the AMB-resistant A. flavus isolate may be also due to mitochondrial abnormalities. However, additional studies are needed. 
In conclusion, our results showed that the AMB-resistant A. flavus isolate presented elevated endogenous ROS levels, an opposite observation to that mediating AMB-resistance in A. terreus. The elevated endogenous ROS contributed to the sensitivity of the AMB-resistant A. flavus isolate to triazoles and echinocandins. However, further investigation is needed to elucidate the causes of elevated endogenous ROS and the resistance mechanism to AMB in A. flavus.

\section{DATA AVAILABILITY STATEMENT}

The original contributions presented in the study are included in the article/supplementary material, further inquiries can be directed to the corresponding author.

\section{REFERENCES}

Ashu, E. E., Korfanty, G. A., Samarasinghe, H., Pum, N., You, M., Yamamura, D., et al. (2018). Widespread amphotericin B-resistant strains of Aspergillus fumigatus in Hamilton. Canada. Infect. Drug Resist. 11, 1549-1555. doi: 10. 2147/IDR.S170952

Belenky, P., Camacho, D., and Collins, J. J. (2013). Fungicidal drugs induce a common oxidative-damage cellular death pathway. Cell. Rep. 3, 350-358. doi: 10.1016/j.celrep.2012.12.021

Blatzer, M., Jukic, E., Posch, W., Schopf, B., Binder, U., Steger, M., et al. (2015). Amphotericin B Resistance in Aspergillus terreus Is Overpowered by Coapplication of Pro-oxidants. Antioxid. Redox. Signal. 23, 1424-1438. doi: 10.1089/ars.2014.6220

Blum, G., Hortnagl, C., Jukic, E., Erbeznik, T., Pumpel, T., Dietrich, H., et al. (2013). New insight into amphotericin B resistance in Aspergillus terreus. Antimicrob. Agents Chemother. 57, 1583-1588. doi: 10.1128/AAC.012 83-12

Blum, G., Perkhofer, S., Haas, H., Schrettl, M., Wurzner, R., Dierich, M. P., et al. (2008). Potential basis for amphotericin B resistance in Aspergillus terreus. Antimicrob. Agents Chemother. 52, 1553-1555. doi: 10.1128/AAC.01280-07

Brown, G. D., Denning, D. W., Gow, N. A., Levitz, S. M., Netea, M. G., and White, T. C. (2012). Hidden killers: human fungal infections. Sci. Transl. Med. 4:165rv113. doi: 10.1126/scitranslmed.3004404

Clinical and Laboratory Standards Institute (CLSI). (2017). Reference Method for Broth Dilution Antifungal Susceptibility Testing of Filamentous Fungi. CLSI Standard M38, 3rd Edn. Wayne: Clinical and Laboratory Standards Institute.

Dekhuijzen, P. N. (2004). Antioxidant properties of N-acetylcysteine: their relevance in relation to chronic obstructive pulmonary disease. Eur. Respir. J. 23, 629-636. doi: 10.1183/09031936.04.00016804

Delattin, N., Cammue, B. P., and Thevissen, K. (2014). Reactive oxygen speciesinducing antifungal agents and their activity against fungal biofilms. Future Med. Chem. 6, 77-90. doi: 10.4155/fmc.13.189

Dringen, R., and Hamprecht, B. (1999). N-acetylcysteine, but not methionine or 2-oxothiazolidine-4-carboxylate, serves as cysteine donor for the synthesis of glutathione in cultured neurons derived from embryonal rat brain. Neurosci. Lett. 259, 79-82. doi: 10.1016/s0304-3940(98)00894-5

Geber, A., Hitchcock, C. A., Swartz, J. E., Pullen, F. S., Marsden, K. E., KwonChung, K. J., et al. (1995). Deletion of the Candida glabrata ERG3 and ERG11 genes: effect on cell viability, cell growth, sterol composition, and antifungal susceptibility. Antimicrob. Agents Chemother. 39, 2708-2717. doi: 10.1128/aac. 39.12.2708

Gray, K. C., Palacios, D. S., Dailey, I., Endo, M. M., Uno, B. E., Wilcock, B. C., et al. (2012). Amphotericin primarily kills yeast by simply binding ergosterol. Proc. Natl. Acad. Sci. U. S. A. 109, 2234-2239. doi: 10.1073/pnas.1117280109

Grazziotin, L. R., Moreira, L. B., and Ferreira, M. A. P. (2018). Comparative Effectiveness and Safety between Amphotericin B Lipid-Formulations: a

\section{AUTHOR CONTRIBUTIONS}

TL, WC, and ZW completed the experiments. TL wrote the manuscript. XY, QW, RL, and WL revised the manuscript. WL conducted the experiments and data analysis. All authors read and approved the manuscript.

\section{FUNDING}

This work was supported by the National Natural Science Foundation of China (grant 81861148028, 81671990, and 81971912 ) and Natural Science Foundation of Guangxi Province of China project Guangxi Innovation Research Team for Fungal Infectious Diseases Prevention and Treatment (grant 2020GXNSFGA238001).

Systematic Review. Int. J. Technol. Assess. Health Care 34, 343-351. doi: 10. 1017/S026646231800034X

Hadrich, I., Makni, F., Neji, S., Cheikhrouhou, F., Bellaaj, H., Elloumi, M., et al. (2012). Amphotericin B in vitro resistance is associated with fatal Aspergillus flavus infection. Med. Mycol. 50, 829-834. doi: 10.3109/13693786.2012.684154

Hao, B., Cheng, S., Clancy, C. J., and Nguyen, M. H. (2013). Caspofungin kills Candida albicans by causing both cellular apoptosis and necrosis. Antimicrob. Agents Chemother. 57, 326-332. doi: 10.1128/AAC.01366-12

Jukic, E., Blatzer, M., Posch, W., Steger, M., Binder, U., Lass-Florl, C., et al. (2017). Oxidative Stress Response Tips the Balance in Aspergillus terreus Amphotericin B Resistance. Antimicrob. Agents Chemother. 61:e00670-17. doi: 10.1128/AAC. 00670-17

Kristanc, L., Bozic, B., Jokhadar, S. Z., Dolenc, M. S., and Gomiscek, G. (2019). The pore-forming action of polyenes: from model membranes to living organisms. Biochim. Biophys. Acta Biomembr. 1861, 418-430. doi: 10.1016/j.bbamem.2018. 11.006

Lambou, K., Lamarre, C., Beau, R., Dufour, N., and Latge, J. P. (2010). Functional analysis of the superoxide dismutase family in Aspergillus fumigatus. Mol. Microbiol. 75, 910-923. doi: 10.1111/j.1365-2958.2009.07024.x

Lee, W., and Lee, D. G. (2018). Reactive oxygen species modulate itraconazoleinduced apoptosis via mitochondrial disruption in Candida albicans. Free Radic. Res. 52, 39-50. doi: 10.1080/10715762.2017.1407412

Margis, R., Dunand, C., Teixeira, F. K., and Margis-Pinheiro, M. (2008). Glutathione peroxidase family - an evolutionary overview. FEBS J. 275, 39593970. doi: 10.1111/j.1742-4658.2008.06542.x

Martel, C. M., Parker, J. E., Bader, O., Weig, M., Gross, U., Warrilow, A. G., et al. (2010a). A clinical isolate of Candida albicans with mutations in ERG11 (encoding sterol 14alpha-demethylase) and ERG5 (encoding C22 desaturase) is cross resistant to azoles and amphotericin B. Antimicrob. Agents Chemother. 54, 3578-3583. doi: 10.1128/AAC.00303-10

Martel, C. M., Parker, J. E., Warrilow, A. G., Rolley, N. J., Kelly, S. L., and Kelly, D. E. (2010b). Complementation of a Saccharomyces cerevisiae ERG11/CYP51 (sterol 14alpha-demethylase) doxycycline-regulated mutant and screening of the azole sensitivity of Aspergillus fumigatus isoenzymes CYP51A and CYP51B. Antimicrob. Agents Chemother. 54, 4920-4923. doi: 10.1128/AAC.00349-10

Mesa-Arango, A. C., Trevijano-Contador, N., Roman, E., Sanchez-Fresneda, R., Casas, C., Herrero, E., et al. (2014). The production of reactive oxygen species is a universal action mechanism of Amphotericin B against pathogenic yeasts and contributes to the fungicidal effect of this drug. Antimicrob. Agents Chemother. 58, 6627-6638. doi: 10.1128/AAC.03570-14

Perlin, D. S. (2015). Mechanisms of echinocandin antifungal drug resistance. Ann. N. Y. Acad. Sci. 1354, 1-11. doi: 10.1111/nyas.12831

Perlin, D. S., Rautemaa-Richardson, R., and Alastruey-Izquierdo, A. (2017). The global problem of antifungal resistance: prevalence, mechanisms, and management. Lancet Infect. Dis. 17, e383-e392. doi: 10.1016/S1473-3099(17) 30316-X 
Posch, W., Blatzer, M., Wilflingseder, D., and Lass-Florl, C. (2018). Aspergillus terreus: novel lessons learned on amphotericin B resistance. Med. Mycol. 56, 73-82. doi: 10.1093/mmy/myx119

Qiao, J., Kontoyiannis, D. P., Wan, Z., Li, R., and Liu, W. (2007). Antifungal activity of statins against Aspergillus species. Med. Mycol. 45, 589-593. doi: 10.1080/13693780701397673

Rudramurthy, S. M., Paul, R. A., Chakrabarti, A., Mouton, J. W., and Meis, J. F. (2019). Invasive Aspergillosis by Aspergillus flavus: epidemiology, Diagnosis, Antifungal Resistance, and Management. J. Fungi. 5:55. doi: 10.3390/ jof5030055

Sanglard, D., Ischer, F., Parkinson, T., Falconer, D., and Bille, J. (2003). Candida albicans mutations in the ergosterol biosynthetic pathway and resistance to several antifungal agents. Antimicrob. Agents Chemother. 47, 2404-2412. doi: 10.1128/aac.47.8.2404-2412.2003

Schmittgen, T. D., and Livak, K. J. (2008). Analyzing real-time PCR data by the comparative C(T) method. Nat. Protoc. 3, 1101-1108. doi: 10.1038/nprot.20 08.73

Sena, L. A., and Chandel, N. S. (2012). Physiological roles of mitochondrial reactive oxygen species. Mol. Cell. 48, 158-167. doi: 10.1016/j.molcel.2012.0 9.025

Shekhova, E., Kniemeyer, O., and Brakhage, A. A. (2017). Induction of Mitochondrial Reactive Oxygen Species Production by Itraconazole, Terbinafine, and Amphotericin B as a Mode of Action against Aspergillus fumigatus. Antimicrob. Agents Chemother. 61:e00978-17. doi: 10.1128/AAC.00978-17

Shibuya, K., Paris, S., Ando, T., Nakayama, H., Hatori, T., and Latge, J. P. (2006). Catalases of Aspergillus fumigatus and inflammation in aspergillosis. Nihon. Ishinkin. Gakkai. Zasshi. 47, 249-255. doi: 10.3314/jjmm.47.249

Shirazi, F., Pontikos, M. A., Walsh, T. J., Albert, N., Lewis, R. E., and Kontoyiannis, D. P. (2013). Hyperthermia sensitizes Rhizopus oryzae to posaconazole and itraconazole action through apoptosis. Antimicrob. Agents Chemother. 57, 4360-4368. doi: 10.1128/AAC.00571-13

Silva, L. N., Oliveira, S. S. C., Magalhaes, L. B., Andrade Neto, V. V., Torres-Santos, E. C., Carvalho, M. D. C., et al. (2020). Unmasking the Amphotericin B Resistance Mechanisms in Candida haemulonii Species Complex. ACS Infect. Dis. 6, 1273-1282. doi: 10.1021/acsinfecdis.0 c00117

Stone, N. R., Bicanic, T., Salim, R., and Hope, W. (2016). Liposomal Amphotericin B (AmBisome $\left({ }^{\circledR}\right)$ ): a Review of the Pharmacokinetics, Pharmacodynamics,
Clinical Experience and Future Directions. Drugs 76, 485-500. doi: 10.1007/ s40265-016-0538-7

Vaezi, A., Fakhim, H., Arastehfar, A., Shokohi, T., Hedayati, M. T., Khodavaisy, S., et al. (2018). In vitro antifungal activity of amphotericin B and 11 comparators against Aspergillus terreus species complex. Mycoses 61, 134-142. doi: 10.1111/ myc. 12716

Vincent, B. M., Lancaster, A. K., Scherz-Shouval, R., Whitesell, L., and Lindquist, S. (2013). Fitness trade-offs restrict the evolution of resistance to amphotericin B. PLoS Biol. 11:e1001692. doi: 10.1371/journal.pbio.10 01692

Warrilow, A. G., Melo, N., Martel, C. M., Parker, J. E., Nes, W. D., Kelly, S. L., et al. (2010). Expression, purification, and characterization of Aspergillus fumigatus sterol 14-alpha demethylase (CYP51) isoenzymes A and B. Antimicrob. Agents Chemother. 54, 4225-4234. doi: 10.1128/AAC.00316-10

Warrilow, A. G. S., Parker, J. E., Price, C. L., Rolley, N. J., Nes, W. D., Kelly, D. E., et al. (2019). Isavuconazole and voriconazole inhibition of sterol 14alpha-demethylases (CYP51) from Aspergillus fumigatus and Homo sapiens. Int. J. Antimicrob. Agents 54, 449-455. doi: 10.1016/j.ijantimicag.2019.0 7.011

Zorov, D. B., Juhaszova, M., and Sollott, S. J. (2014). Mitochondrial reactive oxygen species (ROS) and ROS-induced ROS release. Physiol. Rev. 94, 909-950. doi: 10.1152/physrev.00026.2013

Conflict of Interest: The authors declare that the research was conducted in the absence of any commercial or financial relationships that could be construed as a potential conflict of interest.

Publisher's Note: All claims expressed in this article are solely those of the authors and do not necessarily represent those of their affiliated organizations, or those of the publisher, the editors and the reviewers. Any product that may be evaluated in this article, or claim that may be made by its manufacturer, is not guaranteed or endorsed by the publisher.

Copyright (C) 2021 Liang, Chen, Yang, Wang, Wan, Li and Liu. This is an open-access article distributed under the terms of the Creative Commons Attribution License (CC BY). The use, distribution or reproduction in other forums is permitted, provided the original author(s) and the copyright owner(s) are credited and that the original publication in this journal is cited, in accordance with accepted academic practice. No use, distribution or reproduction is permitted which does not comply with these terms. 